\title{
Microneedle Devices for Drug Delivery
}

\author{
Owen J. Guy ${ }^{1}$, Huma Ashraf ${ }^{3}$, Gareth Blayney ${ }^{1}$, Chris Bolton ${ }^{1,3}$, Connie Eng ${ }^{2}$, Olivia Howells ${ }^{1,2}$ \\ Kerry Roberts ${ }^{3}$, Sanjiv Sharma ${ }^{1}$ \\ ${ }^{1}$ Swansea University \\ United Kingdom \\ ${ }^{2}$ BioMEMS Ltd. \\ ${ }^{3}$ SPTS Technologies
}

Microneedles (MNs) are tiny microscopic needles, much smaller than conventional hypodermic needles, that can be used to deliver pharmaceutical drugs or vaccines through the skin into the body in a minimally invasive manner.

Targeted delivery to the layers just beneath the outer surface of the skin (the stratum corneum) can be used to achieve much more effective drug or vaccine delivery.

Microneedles can be made from a number of materials including, metals, plastics and silicon. MN production is scalable using injection moulding (for polymer MNs) or using silicon processing wafers, normally used in the semiconductor chip industry, for manufacture of arrays of hollow MNs. The pros and cons of different MN materials, fabrication techniques and designs will be reviewed.

The sharpness of the MN tips are critical to their effective skin penetration. A novel "bevelled tip" MN design is presented which allow sthe MNs to penetrate into the skin with lower insertion forces. In addition, hollow MNs have been developed - which can be used to inject significant volumes substances into the skin, compared to coated solid MNs.

A review of vaccines, drugs and other entities that can be injected into skin will be presented, along with a discussion of MNs in diagnostic, therapeutic and cosmetic applications. 